J. Clin. Chem. Clin. Biochem.

Vol. 16,1978 , pp. $359-364$

\title{
Ein Modell zur adsorptiven Verteilung von Schilddrüsenhormonen in der Zelle
}

\author{
Von D. Geiseler und $R$. Wahl
}

Medizinische Universitätsklinik Tübingen

(Eingegangen am 16. September 1977/6. Februar 1978)

Zusammenfassung: Mitochondrien aus Rattenlebern wurden in Lösungen von Cytoplasmaproteinen („Cytosol“) suspendiert $\left(0,5-4,7 \mathrm{~g} / 1\right.$ Gesamtprotein). Die adsorptive Verteilung von 3,5, $3^{\prime}-L$-Trijodthyronin (Konzentrationsbereich 0,04-0,50 nmol/1) zwischen der flüssigen Phase des Cytosols und der festen Phase der Mitochondrien wurde untersucht. Es zeigte sich, daß die Verteilung des Hormons von den Proteinkonzentrationen in beiden Phasen abhängt. Die mathematische Formulierung dieses Verteilungsgleichgewichts stimmt mit den experimentellen Ergebnissen überein und ist auch für Thyroxin gültig.

Die in vitro untersuchten adsorptiven Bindungsvorgänge von Schilddrüsenhormonen an Zellproteine werden in vivo als Glieder einer Transportkette zwischen verschiedenen Zellkompartimenten betrachtet. Die Bedeutung der theoretischen Untersuchung für diesen Transport von Schilddrüsenhormonen innerhalb der Zelle wird diskutiert.

\section{A model for the adsorptive distribution of thyroid hormone in the cell}

Summary: Mitochondria from rat livers were suspended in solutions of cytoplasmic proteins ("cytosol") containing $0.5-4.7 \mathrm{~g}$ total protein per liter suspension. In these suspensions the adsorptive distribution of radioactive $3,5,3^{\prime}-L$ triiodothyronine was studied in the concentration range between 0.04 and $0.50 \mathrm{nmol} / 1$. The experiments show that the distribution of the hormone between the liquid phase of the cytosol and the solid phase of the mitochondria depends mainly on the protein concentrations in both phases. The mathematical formulation of this distribution equilibria which is also applicable to thyroxine corresponded closely with the experimental findings.

The processes of the adsorptive binding of thyroid hormones to cell proteins observed in vitro are thought to represent part of a transport chain that exists between different compartments in vivo. The consequences of these considerations on the transport of thyroid hormones within the cell are discussed.

\section{Einführưung}

Die physiologische Schilddrüsenfunktion unterliegt einem wohlbekannten biologischen Regelkreis. Die intraund extrazelluläre Verteilung der Schilddrüsenhormone im Organismus bildet dabei ein wesentliches Glied einer Informationskette. Der Transport der wichtigsten Hormone Thyroxin $\left(T_{4}\right)$ und Trijodthyronin $\left(T_{3}\right)$ von der Blutbahn zu den Organen erfolgt in erster Linie durch Bindung an die Serumproteine Thyroxin-bindendes Globulin, Thyroxin-bindendes Präalbumin und Albumin. Nach dem Ubertritt in die Zelle werden die Hormone von den löslichen Cytoplasmaproteinen (= Cytosolprotène) und den festen Proteinphasen der subzellulären Partikel aufgenommen. Die Bindung žwischen Schilddrüsenhormonen und Trägerproteinen ist im Serum und in der Zelle zum überwiegenden Teil reversibel und relativ un- spezifisch. Dies geht aus der leichten Verdrängbarkeit der proteingebundenen Hormone durch körpereigene Substanzen oder Medikamente hervor und rechtfertigt die Charákterisierung dieser Bindung ohne nachfolgende metabolische Funktion als „adsorptive Verteilung“ $(1,2,3)$.

Das Endglied des Schilddrüsenhormontransports zwischen den cytoplasmatischen Trägerproteinen und dem metabolischen Wirkort innerhalb der Zellorganellen (Kerne, Mitochondrien, Mikrosomen) stellt der Hormonübertritt von der löslichen Cytosolphase auf die feste Organellenphase dar. Neben den Zellkernen besitzen die Mitochondrien für die Hormonverteilung aufgrund ihres hohen Anteils an bindungsfähigen Proteinen besondere Bedeutung (4). Neuerdings werden sie wieder als Hauptwirkort der Schilddrüsenhormone diskutiert (5). 
In der vorliegenden Arbeit soll am Beispiel der Mitochondrien versucht werden, die biphasische Adsorptionsverteilung mathematisch zu formulieren. Dem hierzu entwickelten Modell liegt die experimentell gefundene Verteilung von $\mathrm{T}_{3}$ zwischen den Cytosol- und Mitochondrienproteinen der Rattenleber zugrunde. Ausgehend von der Annahme eines quasi-stationären Zustandes für die am Adsorptionsvorgang beteiligten Konzentrationen der Hormone und Proteine kann eine quantitative Aussage über die Hormonverteilung formuliert werden.

\section{Methoden}

$200 \mathrm{~g}$ schwere weibliche Sprague-Dawley-Ratten wurden von der vena portae aus mit $37^{\circ} \mathrm{C}$ warmer Vollelektrolytlösung (Biosteril) blutfrei perfundiert. Anschließend wurde mit körperwarmer Saccharoselösung $(0,44 \mathrm{~mol} / \mathrm{l})$ so lange weiter perfundiert, bis die Oberfläche glasig glänzte. Nach Ablaufen der überschüssigen Saccharoselösung wurde die Leber mit dem Skalpell zerkleinert und mit dem losen Stempel im DounceHomogenisator in eiskalter Saccharoselösung homogenisiert. Pro Gramm Leber wurden $4 \mathrm{ml}$ Saccharoselösung und $0,03-0,04 \mathrm{ml}$ einer $0,1 \mathrm{~mol} / 1$ Citronensäurelösung eingesetzt. Der pH-Wert des Homogenats wurde auf 6,2 (bei pH $<6,0$ werden die Mitochondrien irreversibel geschädigt, bei $\mathrm{pH}>6,5$ beginnen sie zu schwellen und geben Proteine ab) eingestellt (6). Im Eisbad $\left(0-4^{\circ} \mathrm{C}\right)$ wurde das Homogenat mit Saccharoselösung verdünnt und bei $675 \mathrm{~g} 20 \mathrm{~min}$ bei $4^{\circ} \mathrm{C}$ zentrifugiert. Das Sediment enthielt unzerstörte Zellen und die Kernfraktion, der Überstand Mitochondrien und Mikrosomen in Cytosol. Aus diesem Überstand ließen sich die festen Mitochondrien bei $13000 \mathrm{~g}\left(10 \mathrm{~min}, 4^{\circ} \mathrm{C}\right)$ abtrennen, die Mikrosomen anschließend bei $25000-30000 \mathrm{~g}\left(30 \mathrm{~min}, 4^{\circ} \mathrm{C}\right)$. Der hierbei anfallende Überstand entsprach unserem Cytosol. Das mitochondrienhal tige Sediment wurde ein Mal mit Saccharoselösung gewaschen, um insbesondere Cy toplasmareste zu entfernen. Anschließend wurden die Mitochondrien wieder in Saccharoselösung resuspendiert ( $6 \mathrm{ml}$ Saccharoselösung zu den Mitochondrien aus $1 \mathrm{~g}$ Leber). Sie waren mit Mikrosomen und nicht näher definierbaren Bruchstiucken anderer Zellorganellen verunreinigt. Die elektronenmikroskopische Kontrolle ergab jedoch, daß dieser Anteil nicht mehr als 10-20\% betrug.

$\mathrm{Zu}$ Suspensionen mit verschiedenen Konzentrationen von Mitochondrien- und Cytoplasmaproteinen (bis $2 \mathrm{~g} / 1$ ) in Saccharoselösung wurden $0,323 \mathrm{nmol} / 1$ [ ${ }^{125} \mathrm{~J}$ ]Trijod thyronin (spezifische Aktivität 740-2220 GBq/g) zugegeben. Die physiologische Relation der Proteinkonzentrationen von Cytosol und Mitochondrien (Verhältnis etwa 1:1 bis 1,6:1) wurde dabei miterfaßt. Nach kurzer Inkubation bei $0-4^{\circ} \mathrm{C}$ wurde die Radioaktivität in den abzentrifugierten und mit Saccharoselösung gewaschenen Mitochondrien gemessen und mit der Radioaktivität aliquoter Teile der Suspension $(=100 \%$-Wert $)$ in Bezug gesetzt. Eine eventuell auftretende Dejodierung des Trijodthyronins konnte daher unberücksichtigt bleiben, zumal auch bei $0-4^{\circ} \mathrm{C}$ gearbeitet wurde. Um möglichst viele Meßwerte bei verschiedenen Proteinkonzentrationen aus demselben Leberpool zu gewinnen, mußte auf Mehrfachbestimmungen verzichtet werden. Der Verlauf der Meßkurven ist bei verschiedenen Leberpools infolge des unterschiedlichen Proteingehalts qualitativ gleichartig. Die Bestimmung der Mitochondrien- und Cytoplasmaproteine erfolgte mit einer modifizierten Methode nach Lowry (7). Als Standard diente Rinderalbumin. Die radioaktiven . Schilddrüsenhormone wurden stichprobenweise chromatographisch auf ihre Reinheit kontrolliert.

\section{Das Modell}

\section{Spezielle Formulierung für zwei Proteinkomponenten}

Für den Transport der Schilddrüsenhormone innerhalb der Zelle sind in erster Linie rein adsorptive Bindungs- vorgänge von Bedeutung. Die Einstellung des Adsorptionsverteilungsgleichgewichtes erfolgt in vitro bei $0-4{ }^{\circ} \mathrm{C}$ innerhalb weniger Sekunden $(1,2)$. Metabolisierende Reaktionen sind Sekundärschritte, die mit relativ viel geringerer Geschwindigkeit ablaufen. Die Kinetik der adsorptiven Hormonverteilung innerhalb des dynamischen Zellstoffwechsels kann deshalb im Sinne einer partiellen steady-state-Approximation formuliert werden: Die schnellen, reversiblen Adsorptionsreaktionen (oder Hormon-Protein-Komplexbildungen) befinden sich gegenüber den langsameren metabolisierenden Folgereaktionen praktisch immer im Gleichgewicht und bilden somit ein geschlossenes Teilsystem. Ein Vergleich der experimentellen in-vitro-Befunde mit den in-vivo-Verhältnissen wird durch diese quasi-stationäre Näherung erst zulässig.

Es soll zunächst die vereinfachende Annahme gemacht werden, daß die verschiedenen Proteinkomponenten innerhalb der hier interessierenden Mitochondrienphase und Cytosolphase sich bezüglich der Adsorption von Schilddrüsenhormon weitgehend ähnlich verhalten und als chemisch einheitliche Reaktionspartner auftreten. Weiterhin wird angenommen, daß̣ die Assoziationskonstanten unabhängig vom Adsorptionsgrad der Proteine bleiben, also keine kooperativen Wechselwirkungen zwischen den Proteineinheiten durch die Hormonbindung ermöglicht werden.

Für die äquimolaren Konzentrationen von Protein und Hormon werden folgende Bezeichnungen gewählt:

Konzentration der festen, adsorptionsfähigen (Mitochondrien-) Proteine $B_{1}$ in Phase $1=\left[B_{1}\right]$;

Konzentration der löslichen, adsorptionsfähigen (Cytosol-) Proteine $\mathrm{B}_{2}$ in Phase $2=\left[\mathrm{B}_{2}\right]$;

Konzentration des Schilddrüsenhormons $A=[A]$;

Konzentrationen der proteingebundenen Schilddrüsenhormone $A B_{1}, A B_{2}=\left[A_{1}\right],\left[A_{2}\right]$ (= äquimolare Konzentrationen der mit Schilddrüsenhormon besetzten Proteine).

Die adsorptive Verteilung eines Schilddrüsenhormons auf beide Phasen ergibt sich einmal aus den unterschiedlichen Affinitäten, die die Proteine zum Hormon besitzen, zum anderen aus dem Konzentrationsverhältnis, in welchem die Proteine vorliegen. Die Proteine $B_{1}$ und $B_{2}$ reagieren mit $A$ unter $B i l d u n g$ von $A B_{1}$ und $A B_{2}$, bis sich Hin- und Rückreaktion das Gleichgewicht halten (Abb. 1). Im Gleichgewicht teilt sich die Gesamtkonzentration an zugesetztem Hormon wie folgt auf:

$$
\left[A_{\text {total }}\right]=[A]+\left[\mathrm{AB}_{1}\right]+\left[\mathrm{AB}_{2}\right]
$$

Ebenso beträgt die Gesamt-Konzentration an adsorptionsfähigen Proteinen:

$$
\begin{aligned}
& {\left[\mathrm{B}_{1 \text { total }}\right]=\left[\mathrm{B}_{1}\right]+\left[\mathrm{AB}_{1}\right]} \\
& {\left[\mathrm{B}_{2 \text { total }}\right]=\left[\mathrm{B}_{2}\right]+\left[\mathrm{AB}_{2}\right]}
\end{aligned}
$$

Aufgrund der experimentell ermittelten Bindungskurven für $T_{3}$ kann nur angenommen werden, daß $\left[A B_{1}\right]$ und $\left[A B_{2}\right]$ gegenüber $\left[B_{1}\right]$ und $\left[B_{2}\right]$ vernachlässigbar klein sind, denn die an Mitochondrien- und Cytosolproteine gebundene Hormonmenge nimmt mit steigender zuge- 


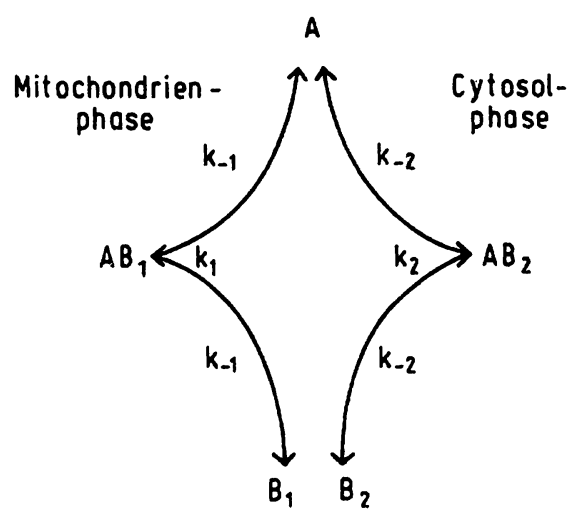

Abb. 1. Kinetisches Schema zur Verteilung eines Schilddrüsenhormons zwischen zwei Proteinphasen.

$\begin{array}{ll}\mathbf{A} & \begin{array}{l}\text { Nicht-proteingebundenes (,freies“) Hormon. } \\ \text { Mitochondrienproteine in der festen Phase 1. }\end{array} \\ \mathbf{B}_{1} & \begin{array}{l}\text { Cytosolproteine in der flüssigen Phase 2. } \\ \mathbf{B}_{2}\end{array} \\ \mathbf{A B}_{1}, \mathbf{A B}_{2} & \begin{array}{l}\text { Proteingebundene Hormone. } \\ \mathbf{k}_{1}, \mathbf{k}_{2}\end{array} \\ \begin{array}{l}\text { Effektive Umsatzkonstanten der Assoziations- } \\ \text { reaktionen. }\end{array} \\ \mathbf{k}_{-1}, \mathbf{k}_{-2} & \begin{array}{l}\text { Effektive Umsatzkonstanten der Dissozia- } \\ \text { tionsreaktionen. }\end{array}\end{array}$

setzter Hormonkonzentration stets linear zu. Es läßt sich bis zu Hormonkonzentrationen von $25 \mathrm{nmol} / \mathrm{l}$, also Konzentrationen, die außerhalb des physiologisch vorkommenden Bereichs liegen (Hormonkonzentration etwa 0,04 bis $0,05 \mathrm{nmol} / \mathrm{l}$; Proteingehalt der festen Mitochondrien 0,66 g/l), keine Absättigung der Mitochondrien-Proteine nachweisen. Dies bedeutet, daß der freie Proteingehalt weit über dem hormongebundenen Teil liegt, so daß in guter Näherung

$$
\left[\mathrm{B}_{1 \text { total }}\right]=\left[\mathrm{B}_{1}\right]
$$

und

$$
\left[B_{2 \text { total }}\right]=\left[B_{2}\right]
$$

gesetzt werden kann.

Für die Umsatzgeschwindigkeiten von $A B_{1}$ und $A B_{2}$ erhält man damit unter Berücksichtigung des Schemas der Abbildung 1 folgende gekoppelte Differentialgleichungen:

$$
\begin{aligned}
\frac{d\left[\mathrm{AB}_{1}\right]}{\mathrm{dt}}= & -k_{-1}\left[\mathrm{AB}_{1}\right]+k_{+1}\left[\mathrm{~B}_{1}\right]\left(\left[\mathrm{A}_{\text {total }}\right]-\right. \\
& \left.-\left[\mathrm{AB}_{1}\right]-\left[\mathrm{AB}_{2}\right]\right) \\
\frac{\mathrm{d}\left[\mathrm{AB}_{2}\right]}{\mathrm{dt}}= & -k_{-2}\left[\mathrm{AB}_{2}\right]+k_{+2}\left[\mathrm{~B}_{2}\right]\left(\left[\mathrm{A}_{\text {total }}\right]-\right. \\
& \left.-\left[\mathrm{AB}_{1}\right]-\left[\mathrm{AB}_{2}\right]\right)
\end{aligned}
$$

Im Gleichgewicht werden die Umsatzgeschwindigkeiten gleich Null, so daß $\left[\mathrm{AB}_{1}\right]$ und $\left[\mathrm{AB}_{2}\right]$ bestimmt werden können. Man erhält:

$$
\begin{aligned}
& {\left[\mathrm{AB}_{1}\right]=\frac{\left[\mathrm{A}_{\text {total }}\right]\left[\mathrm{B}_{1}\right]}{\left[\mathrm{B}_{1}\right]+\frac{k_{-1}}{k_{+1}}+\frac{k_{-1} \mathrm{k}_{+2}}{k_{+1} k_{-2}}\left[\mathrm{~B}_{2}\right]}} \\
& {\left[\mathrm{AB}_{2}\right]=\frac{\left[\mathrm{A}_{\text {total }}\right]\left[\mathrm{B}_{2}\right]}{\left[\mathrm{B}_{2}\right]+\frac{k_{-2}}{k_{+2}}+\frac{k_{-2} \mathrm{k}_{+1}}{k_{+2} \mathrm{k}_{-1}}\left[\mathrm{~B}_{1}\right]}} \\
& \frac{\mathrm{k}_{+1}}{\mathrm{k}_{-1}}=\frac{1}{\mathrm{~K}_{1}}=\text { Assoziationskonstante der Reaktion } \\
& \mathrm{A}+\mathrm{B}_{1} \rightleftarrows \mathrm{AB}_{1} \\
& \frac{\mathrm{k}_{+2}}{\mathrm{k}_{-2}}=\frac{1}{\mathrm{~K}_{2}}=\mathrm{Assoziationskonstante} \mathrm{der} \mathrm{Reaktion}^{\mathrm{A}+\mathrm{B}_{2}} \rightleftarrows \mathrm{AB}_{2}
\end{aligned}
$$

Bildet man die Quotienten $\left[\mathrm{AB}_{1}\right] /\left[\mathrm{A}_{\text {total }}\right]$ und $\left[\mathrm{AB}_{2}\right] /$ [A $\left.A_{\text {total }}\right]$, so erhält man zwei Ausdrücke für das Verhältnis von jeweils gebundenem Hormon pro zugesetztern Hormon. Damit ist ein Zusammenhang zwischen dem Bruchteil des proteingebundenen Hormons und dem Mengenverhältnis des Proteinphasen formuliert.

Zur experimentellen Prüfung dieser Uberlegungen wurden verschiedene Konzentrationen von Mitochondrien- und Cytosolproteinen mit radioaktiv markiertem $\mathrm{T}_{3}$ ins Gleichgewicht gesetzt. Durch Abtrennen der sedimentierten Mitochondrienphase vom Uberstand und Messung der Radioaktivität ergab sich ein Maß für die Konzentration des mitochondriengebundenen $T_{3}$. In zwei Meßreihen wurde diese Hormonmenge bei variablen Konzentrationen an Cytosolproteinen bzw. Mitochondrienproteinen bestimmt. Beide Funktionen $\left[A B_{1}\right] /\left[A_{\text {total }}\right]=f\left(\left[B_{2}\right]\right)$, Parameter $\left[B_{1}\right]$ und $\left[A B_{1}\right] /\left[A_{\text {total }}\right]=g\left(\left[B_{1}\right]\right)$, Parameter $\left[B_{2}\right]$ sollten Gleichung (3) genügen.

Alle Größen in Gleichung (3) und Gleichung (4) sind in molaren Konzentrationseinheiten einzusetzen. Sowohl die mittleren Molekulargewichte der Proteine als auch die Umsetzungsverhältnisse von Proteinen und Hormonen sind unbekannt. Die adsorptiv wirksame Proteinkonzentration $\left[B_{1}\right]$ bzw. $\left[B_{2}\right]$ in der Dimension mol/l ergibt sich daher aus der meßbaren Konzentration $\left[B_{1}^{\prime}\right]$ bzw. $\left[B_{2}^{\prime}\right]$ in $\mathrm{g} / \mathrm{l}$ nach Division durch die Konstante $c_{1}$ bzw. $c_{2}$. Nach Erweiterung mit $c_{1}$ erhält man so für Gleichung (3):

$$
\frac{\left[A B_{1}\right]}{\left[A_{\text {total }}\right]}=\frac{\left[B_{1}^{\prime}\right]}{\left[B_{1}^{\prime}\right]+c_{1} K_{1}+\frac{c_{1} K_{1}}{c_{2} K_{2}}\left[B_{2}^{\prime}\right]}
$$

Damit lassen sich die unbekannten Konstanten $c_{1}, c_{2}$ in $\mathrm{K}_{1}, \mathrm{~K}_{2}$ miteinbeziehen, so daß die Proteinkonzentrationen in der Dimension $\mathrm{g} / \mathrm{l}$ eingesetzt werden können. Die beiden neuen „Assoziationskonstanten“ $1 / K_{1}^{\prime}$, $1 / K_{2}^{\prime}$ erscheinen dann entsprechend in der Dimension $1 / g$. 


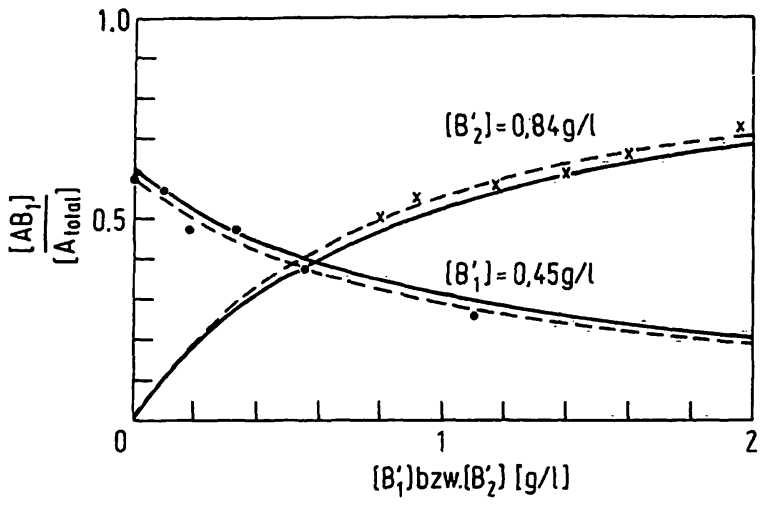

Abb. 2. Trijodthyroninbindung an Rattenleber-Mitochondrien in flüssigem Cytosol in Abhängigkeit von der gemessenen Konzentration an Mitochondrienproteinen (ansteigende Kurven) und der gemessenen Konzentration an Cytosolproteinen (abfallende Kurven).

$\begin{array}{ll}\frac{\left[A B_{1}\right]}{\left[A_{\text {total }}\right]} \quad \begin{array}{l}\text { Bruchteil des mitochondriengebundenen } \\ \text { Hormons }\end{array} \\ {\left[\mathrm{B}_{1}^{\prime}\right]} & \begin{array}{l}\text { Konzentrationen der Mitochondrien- } \\ \text { proteine. } \\ \text { Konzentration der Cytosolproteine. } \\ \text { Meßpunkte der Adsorptionskurve bei } \\ \text { konstantem }\left[\mathrm{B}_{2}^{\prime}\right](=0,84 \mathrm{~g} / \mathrm{l}) .\end{array} \\ \mathbf{X} & \begin{array}{l}\text { Meßpunkte der Desorptionskurve bei } \\ \text { konstantem }\left[\mathrm{B}_{1}^{\prime}\right](=0,45 \mathrm{~g} / \mathrm{l}) .\end{array} \\ - & \begin{array}{l}\text { Ausgleichskurven für die gemessenen } \\ \text { Punkte. }\end{array} \\ & \begin{array}{l}\text { Berechnete Kurven unter Zugrundelegung } \\ \text { der Gleichung (5) mit } \mathrm{K}_{1}^{\prime}=0,27 \mathrm{~g} / 1 \text { und } \\ \mathrm{K}_{2}^{\prime}=0,37 \mathrm{~g} / \mathrm{l} .\end{array}\end{array}$

Die Bestimmung der „Assoziationskonstanten“ aus den Messungen kann durch reziproke Formulierung von Gleichung (5) und lineare Auftragung der Funktion $\left[A_{\text {total }}\right] /\left[\mathrm{AB}_{1}\right]=f\left(\left[\dot{B}_{2}\right]\right)$ erfolgen. Aus Steigung und Ordinatenabschnitt ergeben sich nach Umformung die Konstanten $K_{1}^{\prime}, K_{2}^{\prime}$. Eleganter ist eine direkte; nichtlineare Regression beider Meßreihen. Dabei liefert jede Meßreihe Werte für beide Konstanten, die jeweils untereinander geringfügig differieren. Mit den Mittelwerten von $0,27 \mathrm{~g} / \mathrm{l}$ für $\mathrm{K}_{1}^{\prime}$ und $0,37 \mathrm{~g} / \mathrm{l}$ für $\mathrm{K}_{2}^{\prime}$ stimmen berechnete und gemessene Kurven gut überein (Abb. 2). Für $\mathrm{T}_{4}$ ließen sich ganz analoge Verhältnisse nachweisen (3).

\section{Allgemeine Formulierung}

Die Beziehungen (3) (4) gelten strenggenommen nur, wenn in jeder Proteinphase nur eine chemisch einheitliche Bindungskomponente für die Schilddrüsenhormone enthalten ist. Mitochondrien- und Cytosolphase sind . jedoch komplexe biologische Fraktionen, die mehrere adsorptionsfähige Proteine mit unterschiedlicher HormonAffinität enthalten (8-11). Je geringer die Unterschiede der einzelnen Assoziationskonstanten, desto eher wird der Modellansatz für zwei Komponenten den experimentellen Beobachtungen genügen. Im Folgenden soll nun gezeigt werden, daß die abgeleiteten Beziehungen den
Spezialfall einer allgemeinen Formulierung mit Berücksichtigung von mehr als zwei Proteinkomponenten dàrstellen.

Gleichung (3) und Gleichung (4) erscheineñ wesentlich einfacher, wenn alle Größen in dimensionsloser Form auftreten. Setzt man für

$$
\begin{array}{ll}
\frac{\left[\mathrm{AB}_{1}\right]}{\overline{\left[\mathrm{A}_{\text {total }}\right]}=\alpha_{1}} & \frac{\left[\mathrm{AB}_{2}\right]}{\left[\mathrm{A}_{\text {total }}\right]}=\alpha_{2} \\
\frac{\left[\mathrm{B}_{1}\right]}{\tilde{\mathrm{K}}_{1}}=\beta_{1} & \frac{\left[\mathrm{B}_{2}\right]}{\overline{\mathrm{K}}_{2}}=\beta_{2}
\end{array}
$$

so erhält man für Gleichung (3) und Gleichung (4) viel kürzer:

$$
\begin{aligned}
& \alpha_{1}=\frac{\beta_{1}}{1+\beta_{1}+\beta_{2}} \\
& \alpha_{2}=\frac{\beta_{2}}{1+\beta_{1}+\beta_{2}}
\end{aligned}
$$

$\alpha_{1}$ ist der Bruchteil der von Phase 1 gebundenen Hormonaktivität, ausgedrückt in Abhängigkeit von der reduzierten Konzentration der Komponente 1 in der Phase 1 und der reduzierten Konzentration der Komponente 2 in der Phase 2. Die Summe

$$
\alpha=\alpha_{1}+\alpha_{2}=\frac{\beta_{1}+\beta_{2}}{1+\beta_{1}+\beta_{2}}
$$

gibt den Bruchteil der gesamten gebundenen Hormonmenge in beiden Phasen an.

Bei p Proteinkomponenten, welche alle mit dem gleichen Hormon der Konzentration [ $\left.A_{\text {total }}\right]$ zu reagieren ver-

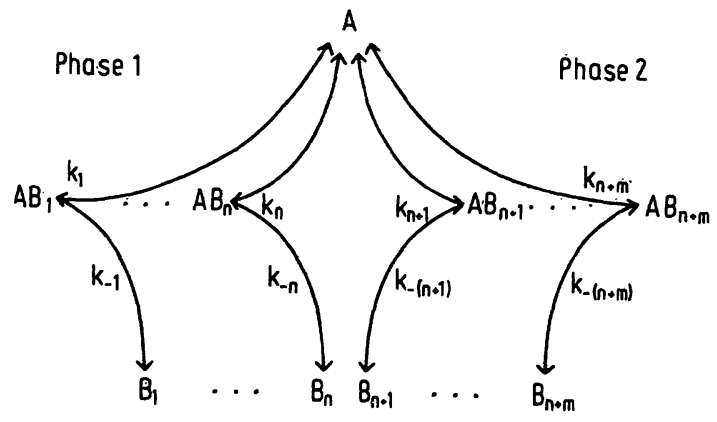

Abb. 3. Kinetisches Schema zur Verteilung eines Schilddrüsenhormons zwischen zwei Proteinphasen mit jeweils mehr als einer Proteinkomponente.
A
Nicht-proteingebundenes (,freies")
$B_{1}$ bis $B_{n}$
$B_{n+1}$ bis $B_{n+m}$ Hormon. Proteinkomponenten in der Phase 2. Proteingebundenes Hormon in beiden
- $k_{1}$ bis $k_{n+m}$ Phasen.
$k_{-1}$ bis $k_{-(n+m)}$
Effektive Umsatzkonstanten der Assoziationsreaktionen.
Effektive Umsatzkonstanten der Dissoziationsreaktionen. 
mögen (Abb. 3), erhält man für die Formulierung des Gleichgewichtes p Gleichungen:

$$
\begin{gathered}
-\alpha_{1}+\beta_{1}\left(1-\sum_{i=1}^{p} \alpha_{i}\right)=0 \\
\vdots \\
-\alpha_{p}+\beta_{p}\left(1-\sum_{i=1}^{p} \alpha_{i}\right)=0
\end{gathered}
$$

Die Umformung ergibt ein inhomogenes Gleichungssystem der $p$ Gleichungen mit $p$ Unbekannten $\alpha_{1}$ bis $\alpha_{p}$. Nach Separation des Lösungsvektors $\underline{\alpha}$ erhält man:

$$
\left[\begin{array}{cccccc}
\left(1+\beta_{1}\right) & \beta_{1} & . & . & \beta_{1} \\
\beta_{2} & \left(1+\beta_{2}\right) & \cdot & \cdot & \beta_{2} \\
\vdots & & & & \vdots \\
\beta_{p} & \beta_{p} & \cdot & \cdot & \cdot\left(1+\beta_{p}\right)
\end{array}\right]\left[\begin{array}{c}
\alpha_{1} \\
\alpha_{2} \\
\vdots \\
\alpha_{p}
\end{array}\right]=\left[\begin{array}{c}
\beta_{1} \\
\beta_{2} \\
\vdots \\
\beta_{p}
\end{array}\right]
$$

Die Lösung für ein $\alpha_{k}$ wird nach Entwicklung der Determinanten und Anwendung der Cramerschen Regel erhalten:

$$
\alpha_{k}=\frac{\beta_{k}}{1+\sum_{i=1}^{p} \beta_{i}}
$$

Bei Bezug auf zwei verschiedene Proteinphasen 1, 2 (in diesem Fall Mitochondrien- und Cytosolphase) mit jeweils $n$ bżw. $m$ Komponenten beträgt der Bruchteil an gebundenem Hormon in beiden Phasen:

Phase 1: $\sum_{i=1}^{n} \alpha_{i}=\frac{\sum_{i=1}^{n} \beta_{i}}{1+\sum_{i=1}^{n} \beta_{i}+\sum_{j=n+1}^{n+m} \beta_{j}}$

Phase 2: $\sum_{j=n+1}^{n+m} \alpha_{j}=\frac{\sum_{j=n+1}^{n+m} \beta_{j}}{1+\sum_{i=1}^{n} \beta_{i}+\sum_{j=n+1}^{n+m} \beta_{j}}$

Dabei bedeuten ạnạlog:

$\alpha_{i}=\frac{\left[A B_{i}\right]}{\left[A_{\text {total }}\right]}: \begin{aligned} & \text { Bruchteil des von der Komponente i } \\ & \text { in der Phạse } 1 \text { gebundenen Hormons }\end{aligned}$ $\alpha_{j}=\frac{\left[\mathrm{AB}_{\mathrm{j}}\right]}{\left[\mathrm{A}_{\text {total }}\right]}: \begin{aligned} & \text { Bruchteil des von der Komponente } \mathrm{j} \\ & \text { in der Phase } 2 \text { gebundenen Hormons }\end{aligned}$ $\beta_{\mathbf{i}}=\frac{\left[\mathrm{B}_{\mathbf{i}}\right]}{\mathrm{K}_{\mathbf{i}}} \quad: \quad \begin{aligned} & \text { Reduzièrte Konzentration der Kom- } \\ & \text { ponente i in der Phase } 1\end{aligned}$ $\beta_{j}=\frac{\left[\bar{B}_{j}\right]}{\bar{K}_{j}} \quad$ Reduzierte Konzentration der Kom$\beta_{\mathrm{j}}=\frac{\mathrm{K}_{\mathrm{j}}}{\grave{\mathrm{K}}_{\mathbf{j}}} \quad$ : ponente $\mathrm{j}$ in der Phase 2
Diese Beziehungen formulieren das Adsorptionsverteilungsgleichgewicht der Hormone zwischen zwei Proteinphasen für eine beliebige Anzahl von Komponenten unter den im letzten Abschnitt angenommenen Voraussetzungen. Bei Kenntnis der „Assoziationskonstanten“ $1 / K_{\mathbf{i}}^{\prime}$ und $1 / K_{j}^{\prime}$ und der Zusammensetzung der Proteinphasen läßt sich die Hormonverteilung berechnen. Einer Bestimmung der Konstanten durch Messung der Verteilung bei zwei Phasen mit je einer reinen Komponente steht prinzipiell nichts im Wege.

\section{Diskussion}

Das hier entwickelte Modell stellt den denkbar einfachsten Ansatz dar, der zur Erklärung der experimentell ermittelten Verteilung von Trijodthyronin zwischen Leberzellmitochondrien und Cytosol möglich ist. Es bestätigt quantitativ die Abhängigkeit der gemessenen Hormonverteilung von den Gesamtproteinkonzentrationen in der löslichen Cytoplasmaphase und der festen Mitochondrienphase. Wie Gleichung (5) zeigt, ändern sich bei Bezug auf die nur hormonbindenden Proteinanteile (experimentell nicht bestimmt) lediglich die Konstanten $\mathbf{K}_{1}^{\prime}, \mathbf{K}_{2}^{\prime}$. Das Modell belegt die Beobachtung, daß bei einem bestimmten Proteinkonzentrationsverhältnis der Anteil an mitochondriengebundenem $\mathrm{T}_{3}$ im untersuchten Konzentrationsbereich $(0,04$ bis $25 \mathrm{nmol} / \mathrm{l})$ konstant bleibt. Für Thyroxin läßt sich die gleiche Gesetzmäßigkeit zeigen (3).

Darüber hinaus erweist sich diese Modellbetrachtung als hinreichend für ein kinetisches Verständnis der Transportvorgänge, bei denen die Bindung an das Trägerprotein überwiegend adsorptiver Natur ist. Wie Abbildung 2 zeigt, liegt immer, auch in Abwesenheit von löslichem Cytoplasmaprotein, ein bestimmter Anteil an nichtmitochondriengebundenem Hormon vor. Dieser Anteil, welcher teilweise frei-diffusibles $T_{3}$ darstellt (im Überstand der reinen Mitochondrienfraktion ohne Cytosolproteine sind zwangsläufig Spuren von löslichen, $T_{3}$-bindenden Proteinen enthalten), könnte bis zu den auf der inneren Mitochondrienmembran liegenden Rezeptoren vordringen, die an den hormonspezifischen Stoffwechsel gekoppelt sind (5). Der Übergang von ,freiem" Hormon in die Mitochondrien hinein wäre ein Glied innerhalb der intrazellulären Transportkette: In dem Maße, wie freies Hormon durch Abbau entzogen wird, werden wieder Bindungsplätze an der Mitochondrienaußenseite frei. Dies bedeutet eine Erhöhung der Menge an bindungsfähigen Mitochondrienproteinen $\left(B_{1}\right)$, wodurch entsprechend dem Verteilungsgleichgewicht gebundenes Hormon $\left(\mathrm{AB}_{2}\right)$ dem löslichen Cytoplasma $\left(B_{2}\right)$ entzogen wird. Für den Ubertritt des Hormons aus dem Cytosol in die Mitochondrien wäre demnach kein aktiver Prozeß erforderlich. Der intramitochondriale Metabolismus dürfte damit letztlich über das hier diskutierte Adsorptionsvertei- 
lungsgleichgewicht an die intra- und extrazellulären Hormonpools gekoppelt sein.

Mitochondrien von Herzmuskelzellen verhalten sich hinsichtlich der Verteilung von $\mathrm{T}_{3}$ und $\mathrm{T}_{4}$ in vitro ebenso wie Lebermitochondrien $(2,3,12)$. Entsprechendes gilt für die Mitochondrien aus Nierengewebe (unveröffentlicht). Mikrosomen haben ein adsorptives Verhalten, das dem von flüssigem Cytoplasma ähnlich ist (2). Als Verunreinigung der Mitochondrienpräparation führen Mikrosomen allenfalls zu einer geringen Parallelverschiebung der Adsorptionskurve (Abb. 2) nach unten.

Eine weitere Bedeutung des Modells könnte darin liegen, daß es durch Einführung zusätzlicher Wechselbeziehungen eine ausbaufähige Arbeitshypothese darstellt. Dies gilt insbesondere für die Beeinflussung des Schilddrüsenhormontransports durch Medikamente. Nicht nur im Serum, sondern gerade in der Zelle können die Adsorptionsverteilungsgleichgewichte durch Medikamente verschoben werden $(2,13)$. Während für die Hormone keine Absättigung der Bindungsplätze im eingesetzten Konzentrationsbereich nachzuweisen war, müssen Sätti- gungserscheinungen bei der Adsorption von Medikamenten wegen der wesentlich höheren Konzentrationen (Faktor $10^{3}-10^{6}$ ) angenommen werden. Ein Medikament kann daher mit den Sčhilddrüsenhormonen um die Bindung am Protein in Konkurrenz treten. Entsprechend dem Verhältnis der Geschwindigkeitskonstanten der Konkurrenzreaktionen kann durchaus eine verstärkte Adsorption der Hormone an die Żellorga- . nellen oder eine Desorption aus diesen resultieren. Die intrazelluläre Verschiebung der Hormonverteilung könnte über die beschriebene Transportkette eine Veränderung des Hormonspiegels im Serum erklären (2, $3,12)$. Diese Bedeutung, die sich zwanglos in den methodischen Rahmen des dargestellten Modells einfügt, kann daher von therapeutischer Konsequenz sein.

\section{Danksagung}

Herrn Prof. Dr. E. Kallee (Isotopen-Abt., Medizinische Universitätsklinik, Tübingen) danken wir für die eingehenden Diskussionen, Frl. $\ddot{H}$. Kaltenbach für die vorzügliche technische Assistenz.

\section{Literatur}

1. Kallee, E. (1966), Acta Isotop. 6, Suppl. 1, 1-95.

2. Wahl, R. \& Kallee, E. (1974), Z. Naturforsch. 29c, 608-617.

3. Wahl, R., Geiseler, D. \& Kallee, E. (1977), Eur. J. Biochem. $80,25-33$.

4. Siebert, G. (1968), in: Handbuch der Allgemeinen Pathologie. Stoffwechsel und Feinstruktur der Zelle I, Bd. 2 (Büchner, F., ed.) pp. 1-237, Springer Berlin.

5. Sterling, K. \& Milch, P. O. (1976), Acta Endocrinol. 84, Suppl. 204, Abstr. 96.

6. Dounce, A. L., Witter, R. F., Monty, K. L., Pate, S. \& Cottone, M. A. (1955), J. Biophys. Biochem. Cytol. 1, 139-153.

7. Bensadoun, A. \& Weinstein, D. (1976), Anal. Biochem. 70, $241-250$.
8. Grimminger, H., Heni, F. \& Kallee, E. (1962), Z. Naturforsch. $17 b, 769-772$.

9. Baudry, M., Clot, J.-P. \& Michel, R. (197.5), Biochimie $57,77-83$.

10. Hamada, S. \& Fukase, M. (1976), in: Thyroid Research (Robbins, J. \& Bravermann, L. E., eds.) pp. 338-341, Excerpta Medica, American Elsevier.

11. Spaulding, S. W. \& Davids, P. J. (1971), Biochim. Biophys. Acta, 229, 279-283.

12. Locher, M., Kaltenbach, H., Wạh, R. \& Kallee, E. (1975), Verh. Dtsch. Ges. Inn. Med. 81, 1564-1567.

13. Gwinup, G. \& Rapp, N. (1975), Am. J. Clin. Pathol. 63, 94-97.

Dr. Dietrich Geiseler Dr. Richard Wahl Medizinische Universitätsklinik D-7400 Tübingen 